\title{
Subwavelength perfect acoustic absorption in membrane-type metamaterials: a geometric perspective
}

\author{
Min Yang, Guancong Ma, Zhiyu Yang, and Ping Sheng* \\ Department of Physics, Hong Kong University of Science and Technology, Clear Water Bay, Kowloon, Hong Kong, China
}

Received 2 September 2015 / Accepted 3 December 2015

\begin{abstract}
Perfect absorption of low frequency sound with subwavelength absorbers has always been a challenge, owing to the difficulty in achieving impedance matching and the inherent weak absorption coefficients of materials at low frequencies. Recently it was shown that when a membrane-type resonator's modes are hybridized through the addition of a thin air-sealed cell with a back reflecting surface, perfect absorption of low frequency acoustic wave can be achieved at a particular tunable frequency. Here we use a geometric perspective, based on the fact that the membrane is very thin and therefore the displacements on both sides of the membrane must be the same, to gain a unified framework for deriving absorption upper bounds as well as for understanding the hybrid resonance and the coherent perfect absorption on the same footing. The latter is another scheme for perfect absorption based on the phase coherence of two counter-propagating waves incident upon the membrane-type resonator. Experiments were carried out to verify some relations predicted by the general framework based on this geometric perspective. Excellent agreement between theory and experiment is seen.
\end{abstract}

Key words: Metamaterials, Decorated membrane resonator, Low-frequency sounds absorption, Coherent perfect absorption, Hybrid resonance metasurface, Geometric perspective.

\section{Introduction}

Even in the 21st century, the absorption of low frequency noise with sub-wavelength absorbers still presents a challenge. This is due to the fact that linear dynamics of dissipative systems dictates the fractional dissipated power to be linearly proportional to the elastic deformation energy [1], while the latter is usually negligible in the low frequency regime. To enhance the dissipation, it is usually necessary to increase the energy density through resonance. However, radiation coupling to resonance is an alternative that can be effective in reducing dissipation. Therefore, the difficulty of subwavelength perfect absorption may be stated as the realization of resonances in open systems with high energy density, but with the high density regions being non-radiative. In previous studies, localized resonances in membrane-type metamaterials [2-7], i.e., decorated membrane resonator (DMR) with tunable weights, have shown efficient sound absorption in the low frequency regime [8]. However, as we shall see below, owing to the geometric constraint, the optimum absorption for a DMR is limited to $50 \%$ of the incident energy if the wave is incident from only one side of the sample [9]. More recently, a subwavelength perfect absorber has been realized by hybridizing DMR's two

\footnotetext{
*e-mail: sheng@ust.hk
}

resonances through the addition of a thin gas layer sealed in a deep-subwavelength cell (with a depth that is $\sim 1 / 133$ of the airborne sound wavelength) [9, 10]. The hybrid resonance is known to have maximum energy density 250 times that of the incoming sound, but radiates out only a negligible amount $(<0.6 \%$ of the incident energy).

In this work, we present a geometric perspective to establish a unified framework for understanding DMR's perfect absorption behaviors. Owing to its subwavelength lateral size, it will be shown that only DMR's surface-averaged normal displacement can be coupled to the radiating modes, with all the rest of the deformation energy couples only to the evanescent modes in air $[2,6,10]$. This makes the DMR's anti-resonance, at which the surface-averaged normal displacement component vanishes and only the local deformation dominates [2, 11], the perfect candidate for subwavelength absorption since the coupling to radiating modes is minimal in the vicinity of antiresonance. However, as the anti-resonance itself is not a resonant state, it cannot couple to the incident wave and hence the absorption at anti-resonance is usually negligible. The solution for this dilemma turns out to be simply to place a reflective wall closely behind the DMR, so as to hybridize DMR's eigenmodes into a new type of hybrid resonances whose frequencies are near DMR's anti-resonance frequencies. While the new 
hybrid resonance resembles the normal displacement profile near DMR's anti-resonance, its maximum normal displacement can be more than an order of magnitude larger. Since most of the large deformation energy cannot be radiated, perfect absorption is the consequence [10]. With the unified framework based on our geometric perspective, we can also easily see the emergence of coherent perfect absorption (CPA) [12-17], which constitutes another scheme for perfect absorption that utilizes two phase-coherent, counter-propagating waves to be incident on the DMR.

In what follows, Section 2 presents an analysis of the special character of the DMR that arises from its subwavelength lateral dimension, and show how that leads naturally to the anti-resonance. The geometric perspective and the derived constraint for DMR's absorption are presented in Section 3. The CPA is shown to be a natural outcome of the geometric framework, verified by numerical simulations in Section 4. The multiple-scattering-based perfect absorption is analyzed in Section 5, which leads to the emergence of DMR's hybrid resonance, presented in Section 6. Section 7 describes the experimental realization of the hybrid resonance. Comparison with theoretical predictions is shown to give excellent agreement.

\section{Decompositiion of DMR's motions and anti-resonance}

An important feature of the DMR is that only its surfaceaveraged normal displacement, $\langle W\rangle$, is radiatively coupled to the acoustic waves in air. Here $W$ denotes the displacement normal to the membrane and \langle\rangle represents surface average. The remaining deformation component $\delta W=W-\langle W\rangle$ can only couple to evanescent waves in air and is therefore nonradiative. This is clear from the Fourier wavevectors $k_{\|}$'s that delineate the lateral spatial pattern of $W$. Since acoustic wavelength $\lambda$ is much larger than the lateral dimension of the DMR, it follows that for the $k_{\|}$components of $\delta W$, we must have $\left|k_{\|}\right|>2 \pi / \lambda$. Owing to the displacement continuity and the wave equation, we have $\left|k_{\|}\right|^{2}+k_{\perp}^{2}=(2 \pi / \lambda)^{2}$ for waves in air, where $k_{\perp}$ denotes the wavevector component normal to the membrane. It follows that the $\delta W$ component couples only to the evanescent waves as its associated $k_{\perp}$ must be imaginary. In contrast, because the $k_{\|}$components for $\langle W\rangle$ have a distribution that peaks at $k_{\|}=0$, it can couple to the radiation modes. It will be seen in what follows that the deformation component $\delta W$ is mainly responsible for subwavelength total absorption, whereas the role of $\langle W\rangle$ in hybrid resonance is to impedance match with the incident wave so that no reflection is generated.

DMR's responses under external pressures $p$ are described by its Green function $G$. For the surface averaged component we have $\langle W\rangle=\langle G\rangle\langle p\rangle$. Here the relevant Green function has an explicit expression based on DMR's eigenstates $W_{n}$ given by $[6,11]$,

$$
\langle G\rangle=\sum_{n} \frac{\left|\left\langle W_{n}\right\rangle\right|^{2}}{\rho_{n}\left(\omega_{n}^{2}-\omega^{2}-2 i \omega \beta_{n}\right)},
$$

where $\rho_{n} \equiv \int_{\Omega} \rho\left|W_{n}\right|^{2} \mathrm{~d} V$ is a parameter related to the displacement-weighted mass density for the DMR's $n$th eigenmode at angular eigen-frequency $\omega_{n}, \rho$ is the local density, and $\Omega$ denotes DMR's volume. We denote the viscosity coefficient of the membrane as $\eta$. The dissipation coefficient $\beta_{n}$ in equation (1) is defined by

$$
\beta_{n}=\int_{\Omega} \frac{\eta\left|\nabla\left(\delta W_{n}\right)\right|^{2} \mathrm{~d} V}{2 \rho_{n}} .
$$

It is sometimes convenient to use surface impedance $Z \equiv\langle p\rangle /\langle\dot{W}\rangle$ to characterize DMR's reflection and transmission. Here the over-dot represents time derivative, thus $Z=$ $i /(\omega\langle G\rangle)$ for harmonic motions with angular frequency $\omega$, i.e., $Z$ and $G$ are inversely related.

Equation (1) has a typical Lorentzian form with the real part changing sign below and above the resonant frequency $\omega_{n}$, with a decreasing magnitude way from $\omega_{n}$. For an incident wave frequency $\omega$ that is in-between the two eigen-modes, both modes will be excited but in opposite phases. At a particular in-between frequency the net surface-average component can vanish, i.e., $\langle W\rangle \sim 0$ for a weakly dissipative system (small $\beta_{n}$ ). At this frequency the resonator is mostly decoupled from external waves, and it is denoted the "anti-resonance" frequency. In contrast, the deformation components $\delta W$ of the two resonant modes do not cancel. Hence in the vicinity of the anti-resonance we have the condition $\sqrt{\left\langle(\delta W)^{2}\right\rangle}>\langle W\rangle$. It follows that if a method can be found to create a resonance near the anti-resonance frequency, then the DMR can be an efficient absorber since on the one hand it can very effectively couple to the incident wave, but on the other hand it can have a deformation component of normal displacement that is much larger than the surface-averaged component, as necessitated by the resonance frequency being close to the anti-resonance frequency. Below we show this to be indeed possible.

\section{Geometric constraint on DMR's absorption}

In this section we focus on the implications that follow from the fact that the membrane is very thin and hence the air displacements on two sides of the membrane must be the same.

Consider two counter-propagating incoming waves incident from two sides, acting on the DMR with complex pressure amplitudes $p_{-}^{i}$ and $p_{+}^{i}$. After scattering, they are converted into two outgoing waves with complex pressure amplitudes $p_{-}^{o}$ and $p_{+}^{o}$. Here the subscript " $-(+)$ " denotes the left (right)-hand side region and the superscript " $i(o)$ " stands for incoming (outgoing) waves. Notice that the sound's energy flux in air, $\quad j=\int_{0}^{2 \pi} \operatorname{Re}\left(p e^{-i \omega t}\right) \operatorname{Re}\left(\dot{W} e^{-i \omega t}\right) d(\omega t) /(2 \pi)=p \dot{W} / 2=$ $\pm p^{2} /\left(2 Z_{0}\right)$, is opposite in directions for incident and outgoing waves. Here $Z_{0}$ denotes the air impedance. Therefore, the air's surface-averaged normal displacement velocities on two sides of DMR are given by

$$
\begin{aligned}
& \left\langle\dot{W}_{-}\right\rangle=\frac{2 j_{-}^{i}}{p_{-}^{i}}+\frac{2 j_{-}^{o}}{p_{-}^{o}}=\frac{1}{Z_{0}}\left(p_{-}^{i}-p_{-}^{o}\right), \\
& \left\langle\dot{W}_{+}\right\rangle=\frac{2 j_{+}^{i}}{p_{+}^{i}}+\frac{2 j_{+}^{o}}{p_{+}^{o}}=\frac{1}{Z_{0}}\left(p_{+}^{i}-p_{+}^{o}\right) .
\end{aligned}
$$


Since the thickness of the membrane is negligible, we must have $\left\langle\dot{W}_{-}\right\rangle=\left\langle\dot{W}_{+}\right\rangle$, i.e., there is no relative motion between the two sides of the membrane. That immediately implies that the mean complex pressure amplitude (MCPA), $\bar{p}$, is conserved before and after the scattering:

$$
\bar{p}=\frac{1}{2}\left(p_{-}^{i}+p_{+}^{i}\right)=\frac{1}{2}\left(p_{-}^{o}+p_{+}^{o}\right) .
$$

Conservation of MCPA implies that a portion of the incoming energy has to be preserved from dissipation. This can be made clear by direct analogy to the two (equal-mass) particles scattering in classical mechanics. If we regard the $p$ 's as momenta of the two particles before and after the collision, then equation (4) expresses the fact that the center of mass momentum is always conserved before and after the collision. For wave incident from one-side, like the collision between a moving particle and a stationary one, there is half of the incoming energy associated with the MCPA (the center of mass energy), which has to be conserved and hence cannot be dissipated. Therefore the absorption coefficient $A$ cannot exceed $A=50 \%$.

\section{Coherent perfect absorption (CPA)}

From the above perspective, a natural way to achieve perfect absorption in DMR is to make $\bar{p}=0\left(p_{-}^{i}=-p_{+}^{i}\right)$, i.e., the CPA scenario (see Figure 1c). For perfect absorption, $p_{-}^{o}=p_{+}^{o}=0$, so that the net pressure applied on DMR is given by $\left\langle p_{\text {tot }}\right\rangle=p_{-}^{i}-p_{+}^{i}=2 p_{-}^{i}$. Since the surface-averaged normal velocity $\langle\dot{W}\rangle$ must be equal to that of air, $\left\langle\dot{W}_{-}\right\rangle$, thus $\langle\dot{W}\rangle=p_{-}^{i} / Z_{0}$. In other words, the CPA requirement boils down to $Z=\left\langle p_{\text {tot }}\right\rangle /\langle\dot{W}\rangle=2 Z_{0}$. Since $Z_{0}$ is a real number, hence in accordance with equation (1), to satisfy the CPA requirement a DMR must be in resonance so that its impedance $Z=i /(\omega\langle G\rangle)$ is real.

To demonstrate the CPA, we simulate a DMR mounted on an aluminum frame with an area of $60.8 \mathrm{~cm}^{2}$ (Figure 1c). The DMR consists of a circular rubber membrane that is $19 \mathrm{~mm}$ in radius and $0.2 \mathrm{~mm}$ in thickness. The edge of the membrane is fixed on the aluminum plate, and a $371 \mathrm{mg}$ circular steel platelet with $5 \mathrm{~mm}$ radius is attached to the center of the membrane. Numerical simulations were carried out by using COMSOL Multiphysics (with material parameters shown in Ref. [11]). The first eigenmode $W_{1}$ of this DMR is found to be located at $\omega_{1}=2 \pi \times 212.3 \mathrm{~Hz}$ as shown in Figure 1d. With $\eta=0.042$, the impedance of this DMR at $\omega_{1}$ is given by equation (1) as $Z=2.004 Z_{0}$. This value satisfies the CPA requirement very well.

Since the CPA requirement coincides with the $50 \%$ maximum absorption condition for one-sided incident wave $[16,18]$, we first introduce sound wave from one side with normalized intensity, and record the associated reflection $R$ (black) and transmission $T$ (blue) as shown in Figure 1a. An almost $50 \%$ absorption peak for $A=1-|R|^{2}-|T|^{2}$ is confirmed at the first resonance frequency (denoted by blue dash-dotted line). We then introduce another sound incoming from the opposite direction with the same intensity but opposite phase

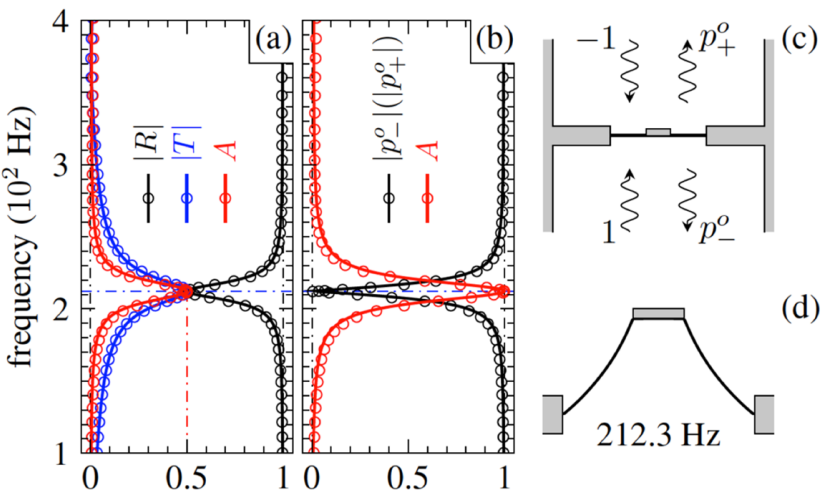

Figure 1. CPA realized by DMR. (a) The reflection (black), transmission (blue), and absorption (red) coefficients for the DMR under one-side incident. (b) The scattering (black) and absorption (red) coefficient under the CPA scenario, i.e., when two beams of sounds come from both sides with the same amplitude but opposite phase as shown in (c). The solid lines are from theoretical calculation based on the eigenmode in (d) and the open circles from numerical simulations. (c) The schematic illustration of the CPA scenario. (d) The simulated cross-sectional profile of the DMR's 1st resonance that satisfies the CPA requirement.

(Figure 1c). As shown in Figure 1b (black), for $\bar{p}=0$, the two scattered waves satisfy $p_{-}^{o}=-p_{+}^{o}$, so that $\left|p_{-}^{o}\right|=\left|p_{+}^{o}\right|$. At the resonance frequency, the relevant absorption coefficient $A=2-\left|p_{-}^{o}\right|^{2}-\left|p_{+}^{o}\right|^{2}$ (red) is seen to reach almost perfect absorption ( 0.999915). The solid curves in Figures 1a and $1 \mathrm{~b}$ are predictions from the impedance for each frequency based on the eigenmode shown in Figure 1d, while the open circles are results of the direct numerical simulation for the scattering process. Excellent agreement is seen.

Although CPA is an efficient scheme for perfect absorption, the required counter-propagating control wave is inconvenient in practice. In what follows, we will show a different strategy for realizing perfect absorption without the control wave.

\section{Total absorption through multiple scatterings}

Although the MCPA energy in the incoming waves has to be conserved in a single scattering event, its complete dissipation is still potentially possible through multiple scatterings. The outgoing MCPA energy after each scattering can serve as the incoming energy for the subsequent scatterings. In the following, we use the geometric perspective to analyze the multiple-scattering process, with the aim of deriving the perfect absorption condition.

In the air layer between the membrane and the reflecting surface, the outgoing wave after $m$ th scattering from the membrane, $p_{+}^{o_{m}}$, is reflected by the wall, and becomes the incoming wave $p_{+}^{i_{m+1}}$, for the $(m+1)$ th scattering. These multiply scattered waves can be superposed to form two counter-propagating waves: $p_{+}^{i}=\sum_{m=1}^{\infty} p_{+}^{i_{m}}$ and $p_{+}^{o}=\sum_{m=0}^{\infty} p_{+}^{o_{m}}$. Because the reflecting wall presents a velocity node, these two wave components in front of the reflecting wall must be equal in 


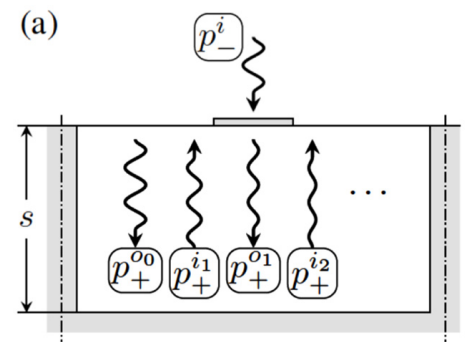

(b)

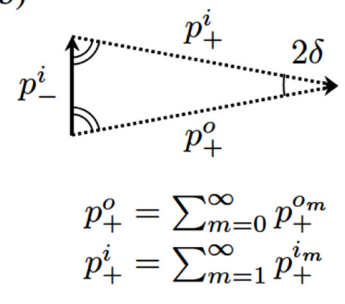

Figure 2. Schematic illustration of the multiple-scattered waves that can lead to perfect absorption on a DMR, with a reflecting wall placed a small distance $s$ behind it. (a) When the incoming wave energy has been totally absorbed, there should be no reflections, i.e., all the scatterings on the incident side cancel each other so that $p_{-}^{o} \exp \left(-i k_{0} z\right)=\sum_{m=0}^{\infty} p_{-}^{o_{m}} \exp \left(-i k_{0} z\right)=0$. Incoming wave $p_{-}^{i} \exp \left(-i k_{0} z\right)$ generates only two net counter-propagating wave components $p_{+}^{o} \exp \left(-i k_{0} z\right)=\sum_{m=0}^{\infty} p_{+}^{o_{m}} \exp \left(-i k_{0} z\right)$ and $p_{2}^{i} \exp \left(-i k_{0} z\right)=\sum_{m=1}^{\infty} p_{+}^{i_{m}} \exp \left(-i k_{0} z\right)$, in the air layer between the DMR (at $z=0$ ) and the reflecting wall. Here the dot-dash lines stand for the location at which the periodic boundary condition is applied. (b) Due to the MCPA conservation, the three complex pressure amplitudes must form an isosceles triangle whose two equal sides are slanted toward each other with an angle given by $2 \delta=2 k_{0} s$.

magnitude and differ in phase by $2 \delta=2 k_{0} s$ at the position of membrane so as to cancel exactly, i.e.,

$$
\left|p_{+}^{i}\right|=\left|p_{+}^{o}\right|, \quad p_{+}^{i} / p_{+}^{o}=\exp (2 i \delta) .
$$

Here $k_{0}$ denotes the sound's wavevector in air. As the MCPA conservation is valid for each scattering, it also holds for their superpositions. Therefore, $\left(p_{-}^{i}+p_{+}^{i}\right) / 2=\left(p_{-}^{o}+\right.$ $\left.p_{+}^{o}\right) / 2$, where $p_{-}^{o}=\sum_{m=0}^{\infty} p_{-}^{o_{m}}$ is the superposition for outgoing waves on the incident side after each scattering. By assuming that the incoming energy is completely absorbed by the DMR, there will be no reflection on the incident side, and $p_{-}^{o}=0$. Thus the conservation of MCPA, $p_{-}^{i}=p_{+}^{o}-p_{+}^{i}$, may be represented by a phasor-diagram, shown in Figure $2 \mathrm{~b}$, which is an isosceles triangle with $p_{+}^{i}$ and $p_{+}^{o}$ being the two equal sides and $p_{-}^{i}$ being the base. It is clear from the diagram that $p_{+}^{i}+p_{+}^{o}$, which is the backside side pressure on the DMR, is perpendicular to $p_{-}^{i}$ and hence differs from it by a phase of $\pi / 2$. Therefore $p_{+}^{i}+p_{+}^{o}=i p_{-}^{i} \cot \delta$. Since the surfaceaveraged velocity is given by $\langle\dot{W}\rangle=\left\langle\dot{W}_{-}\right\rangle=p_{-}^{i} / Z_{0}$, the total pressure applied to the DMR, $\left\langle p_{\text {tot }}\right\rangle=p_{-}^{i}-\left(p_{+}^{i}+p_{-}^{o}\right)$, is given by $\left\langle p_{\text {tot }}\right\rangle=p_{-}^{i}(1-i \cot \delta)$. It follows that the impedance condition of the DMR for achieving total absorption is given by

$$
Z=\left\langle p_{\text {tot }}\right\rangle /\langle\dot{W}\rangle=Z_{0}(1-i \cot \delta)
$$

For $\delta=\pi / 2$, we have $Z=Z_{0}$, i.e., impedance matching to air. This occurs at cell's "drum" resonances when $s=\lambda / 4$. However, for an air layer thinner than a quarter-wavelength, $\delta \rightarrow 0$, and the required imaginary part of $Z$ approaches $-i \infty$. Since the impedance of the whole structure is the addition of the two serial impedances of the DMR and the sealed cell behind it, perfect absorption requires the net impedance of the composite structure $Z_{c}$ to satisfy the condition $Z_{c}=Z_{0}$, i.e., impedance matched to air. In this context the imaginary part of $Z$ in equation (5) is noted to just cancels the large (positive) imaginary part of the thin air layer, so that $\operatorname{Im}\left(Z_{c}\right)=0$. But can the condition as stated by equation (5) be satisfied by the DMR, especially the large negative imaginary part of the impedance? It turns out that the DMR's imaginary part of impedance diverges in the vicinity of anti-resonance frequency, as shown below. Hence impedance matching of our composite structure can indeed occur, but only at a frequency close to the anti-resonance condition. Also, associated with $\operatorname{Im}\left(Z_{c}\right)=0$ is that the Green function of the composite structure, $\left\langle G_{c}\right\rangle=$ $i /\left(\omega Z_{c}\right)$, has a peak in its imaginary part. This is clear since

$$
\operatorname{Im}\left\langle G_{c}\right\rangle=\frac{1}{\omega} \frac{\operatorname{Re}\left(Z_{c}\right)}{\operatorname{Re}\left(Z_{c}\right)^{2}-\operatorname{Im}\left(Z_{c}\right)^{2}},
$$

so that $\operatorname{Im}\left\langle G_{c}\right\rangle$ reaches a peak when $\operatorname{Im}\left(Z_{c}\right)=0$. A peak in the imaginary part of the Green function signifies a resonance mode.

In what follows, we show that a new hybrid resonance of the composite structure precisely fits the required profile deduced from our analysis.

\section{Hybrid resonance and total absorption}

In order to see the emergence of the hybrid mode, for simplicity we consider the normal displacements of only two relevant eigen-modes, $W_{1}$ and $W_{2}$ (see Figure $3 \mathrm{~b}$ ). The surface-averaged Green function of the DMR in equation (1) can be written as

$$
\langle G\rangle \simeq \sum_{n=1}^{2} \frac{\left|\left\langle W_{n}\right\rangle\right|^{2}}{\rho_{n}\left(\omega_{n}^{2}-\omega^{2}\right)}+2 i \beta \sum_{n=1}^{2} \frac{\left|\left\langle W_{n}\right\rangle\right|^{2} \omega}{\rho_{n}\left(\omega_{n}^{2}-\omega^{2}\right)^{2}},
$$

where $\beta$, the averaged coefficient of $\beta_{1}$ and $\beta_{2}$, is taken to be a small quantity so that the dimensionless $\beta / \omega \ll 1$. In anticipation that the hybrid resonance occurs near the anti-resonance, we expand equation (6) in the vicinity of DMR's anti-resonance frequency $\tilde{\omega}$, at which $\operatorname{Re}(\langle G\rangle)=0$. The expansion can be written as $\langle G\rangle \simeq 2 \Xi(i \beta-\Delta \omega)$, where

$$
\Xi \equiv \sum_{n=1}^{2} \frac{\left|\left\langle W_{n}\right\rangle\right|^{2} \tilde{\omega}}{\rho_{n}\left(\omega_{n}^{2}-\tilde{\omega}^{2}\right)^{2}} \text {, and } \Delta \omega \equiv \tilde{\omega}-\omega .
$$

From the inverse relationship between the Green function and impedance, the associated $Z$ is given by

$$
Z=\frac{1}{2 \tilde{\omega} \Xi} \frac{\beta-i \Delta \omega}{\beta^{2}+\Delta \omega^{2}} .
$$

From equation ( 7 ) it is clear that if $\beta=0$, then the imaginary part of the impedance diverges negatively when $\Delta \omega$ approaches zero from the positive side. When $\beta$ is finite but small, then the imaginary part of the impedance can be as large in magnitude as $-1 /(4 \tilde{\omega} \Xi \beta)$, at a frequency below $\tilde{\omega}$ such that $\Delta \omega=\beta$. Therefore, perfect absorption can indeed occur for a DMR backed by a reflecting surface. It is noted that the perfect 

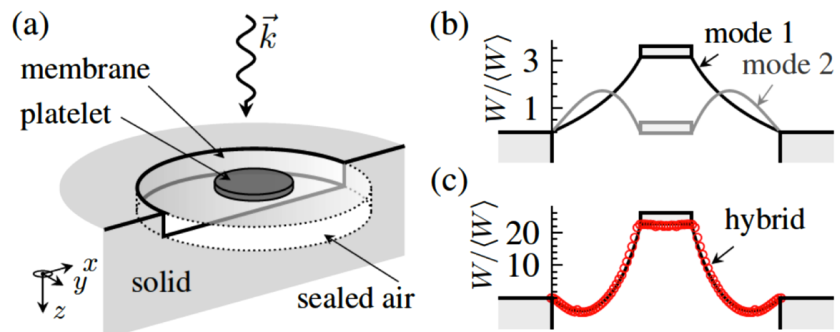

(c)

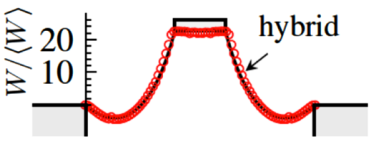

Figure 3. (a) Schematic illustration of the unit cell's component and geometry of the hybrid resonator. (b) Schematic cross-sectional illustration of the two lowest frequency eigen-modes of the DMR, plotted in reference to the same phase of incident wave, with $W$ being the normal displacement of membrane, normalized to its surface average component $\langle W\rangle$. (c) Displacement profile of the DMR's hybrid mode at $152 \mathrm{~Hz}$, which is clearly a superposition of the two in (b), but with an almost an order of magnitude larger amplitude. However, such large amplitude oscillations are only coupled to the non-radiative evanescent waves. Experimentally measured results (symmetrically plotted from data along the radial direction on one side) are shown in red circles while the simulated results are in black curves. Excellent agreement is seen.

absorption actually occurs under the condition of a small dissipative coefficient $\beta$.

Equating equations (5) and (7) yields two equations for the condition of total absorption:

$$
\begin{gathered}
\frac{1}{2 \tilde{\omega} \Xi} \frac{\beta}{\beta^{2}+\Delta \omega^{2}}=Z_{0}, \\
\frac{1}{2 \tilde{\omega} \Xi} \frac{\Delta \omega}{\beta^{2}+\Delta \omega^{2}}=Z_{0} \cot \delta .
\end{gathered}
$$

Although the dissipation coefficient $\beta$ is not easily adjustable, this total absorption condition is still robust since we have two other parameters, $s$ and $\Delta \omega$, which can be easily tuned experimentally to satisfy equation (8). Notice that the solution of equation (8) occurs close to the anti-resonance $(\Delta \omega \rightarrow 0)$ (Figure 4a). As the hybrid mode frequency is between the two DMR modes, its displacement profile is necessarily the linear superposition of the two DMR eigen-modes. It therefore resembles the profile of membrane motions at the vicinity of the anti-resonance frequency. In particular, the magnitudes of the two DMR modes, $\left\langle W_{1}\right\rangle$ and $\left\langle W_{2}\right\rangle$, must be comparable here - namely, $\left\langle W_{1}\right\rangle /\left\langle W_{2}\right\rangle \simeq 1$. However, impedance matching implies $\left\langle W_{1}\right\rangle-\left\langle W_{2}\right\rangle \simeq W_{s}$, the air displacement of incoming sounds. These two conditions can be satisfied only when $\left\langle W_{1,2}\right\rangle \gg W_{s}$, so that $1-\left\langle W_{2}\right\rangle /\left\langle W_{1}\right\rangle=W_{s} /\left\langle W_{1}\right\rangle$ is a small number. In other words, the hybrid resonance satisfies the condition $\sqrt{\left\langle(\delta W)^{2}\right\rangle}>\langle W\rangle$, owing to its proximity to the antiresonance frequency.

\section{Experimental realization}

Experiments reported in reference [10] have corroborated the existence of the hybrid resonance with perfect absorption

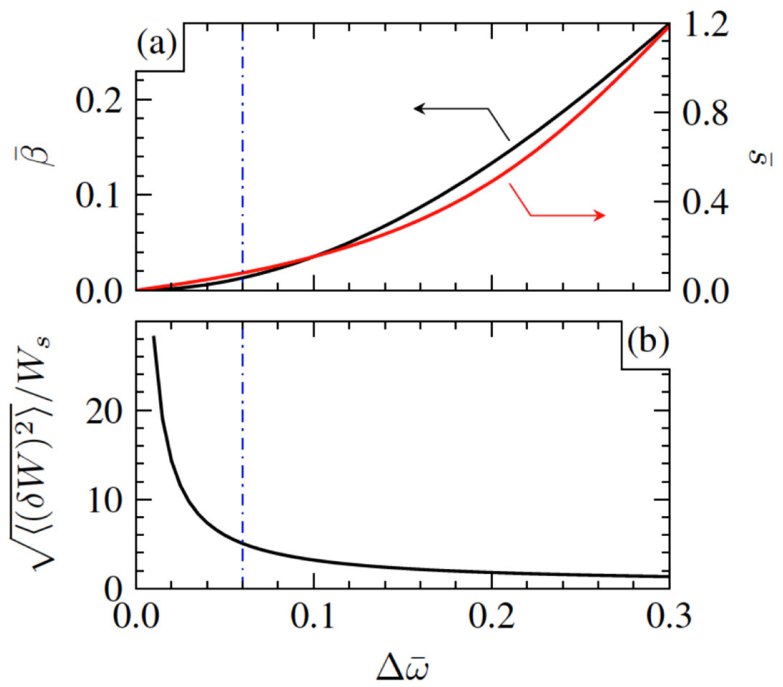

Figure 4. Relationships between different parameters at the hybrid resonant frequency with perfect absorption. (a) Dimensionless dissipation parameter $\bar{\beta}$ (black curve) and dimensionless thickness $\bar{S}$ (red curve) of the sealed air layer, plotted as functions of the dimensionless relative frequency location $\Delta \bar{\omega}$ of the hybrid resonance. (b) Deformation component $\sqrt{\left\langle(\delta W)^{2}\right\rangle}$ of the normal displacement at the hybrid resonance frequency, plotted as a function of $\Delta \bar{\omega}$. Impedance matching means that the surfaceaveraged displacement has to be equal to that of the incident sound - that is, $\langle W\rangle=\left\langle W_{1}\right\rangle-\left\langle W_{2}\right\rangle=W_{s}$. Proximity to the anti-resonance $\tilde{\omega}$ also means that $\left\langle W_{1}\right\rangle \simeq\left\langle W_{2}\right\rangle$. These two conditions imply a large $W$ compared with its surface average, and account for its large variance component of the deformation $\sqrt{\left\langle(\delta W)^{2}\right\rangle}$. In particular, the small dissipation coefficient and the subwavelength scale dovetail with the large $W$ in a self-consistent manner so as to achieve total absorption. The dot-dashed blue lines in the two figures indicate the parameter values relevant to the experimental results shown in Figure 5.

capability. By placing an aluminum-reflecting wall behind a DMR with a distance $s$ (Figure 3a), an absorption peak with magnitude greater than $99 \%$ was observed at $152 \mathrm{~Hz}$ (Figure 5c). In this case the DMR comprises a $90 \mathrm{~mm}$-wide circular elastic membrane decorated by a $800 \mathrm{mg}$ platelet with a radius of $10 \mathrm{~mm}$. The value of $s$ is $21.7 \mathrm{~mm}$, which is inferred from the experimental data of $17 \mathrm{~mm}$ of SF6 gas, as the effects of the two thin gas layers are identical if their thicknesses are scaled linearly with their adiabatic indices (SF6 has an adiabatic index of 1.098 , compared to 1.4 for air). Note here that the air layer is in the deep-subwavelength regime, $s \simeq \lambda / 104$, where $\lambda=2.26 \mathrm{~m}$ is the wavelength of $152 \mathrm{~Hz}$ sound in air.

Numerical simulations by COMSOL Multiphysics (with material parameters shown in Ref. [11]) are based on the two relevant eigen-modes for the DMR as shown in Figure $3 \mathrm{~b}$. The values of the relevant parameters are $\Xi=1.268 \times 10^{-9} \mathrm{~m}^{2} \mathrm{~s}^{3} / \mathrm{kg}$ and $\tilde{\omega}=2 \pi \times 162.3 \mathrm{~Hz}$. In Figure $4 \mathrm{a}$ we plot the dimensionless $\bar{\beta}=\beta / \tilde{\omega}$ (black curve) and $\bar{s}=s /\left(\gamma p_{0} Z_{0}^{-1} \tilde{\omega}^{-1}\right)$ (red curve) that satisfy condition (8) as a function of $\Delta \bar{\omega}=\Delta \omega / \tilde{\omega}$. Here $\gamma=1.4$ is the adiabatic index of air and $p_{0}$ is the atmospheric pressure. It is clear that 


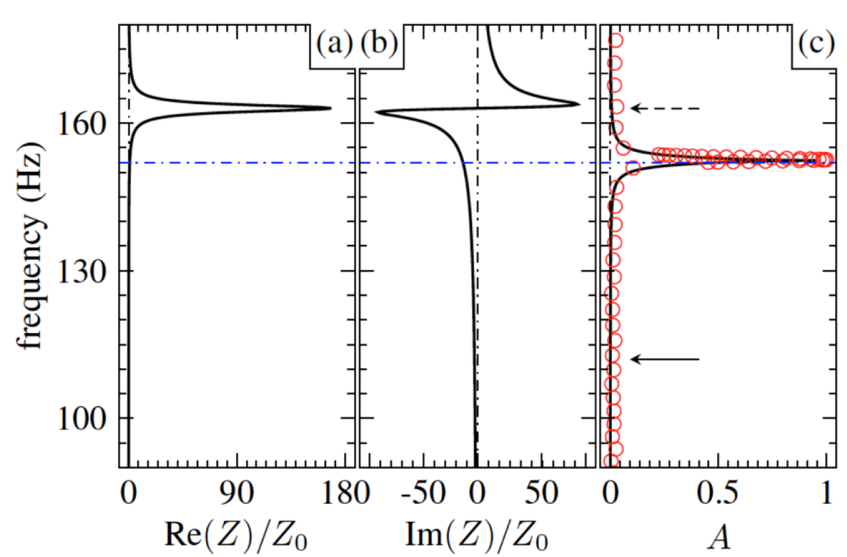

Figure 5. (a), (b) The real and imaginary parts of the DMR's normalized surface impedance $Z / Z_{0}$, where $Z_{0}$ denotes the impedance of air, plotted as a function of frequency. They are seen to satisfy the total absorption requirements given by equation (5) at the hybrid resonance frequency, indicated by the intersection of the black curves with the blue dot-dashed line. Total absorption is confirmed experimentally, shown in (c). A sharp absorption peak, reaching 0.994 , is seen. The solid and dashed arrows indicate the first eigenmode and the anti-resonance frequency of the DMR, respectively. Experimental results are shown by the red circles, and the black curves denote the theoretical predictions.

for the hybrid resonant frequency close to anti-resonance, we have $\Delta \bar{\omega} \rightarrow 0$, and the associated dissipation coefficient $\bar{\beta}$ and air layer thickness $\bar{s}$ are both much smaller than 1. In particular, for the value $\Delta \bar{\omega}=0.06$, which corresponds with the observed total absorption at $2 \pi \times 152 \mathrm{~Hz}$ (indicated by blue dot-dashed line), one can evaluate the dissipation coefficient $\bar{\beta}=0.00502(\beta=5.14 \mathrm{~Hz})$, and predict the thickness of air layer to be $\bar{s}=0.0772(s=21.9 \mathrm{~mm})$. The latter is noted to be in excellent agreement with the experimental value of $s=21.7 \mathrm{~mm}$.

The real and imaginary parts of surface impedance of the DMR, given by equation (7) as a function of frequency, are shown in Figures 5a and 5b, respectively. At the total absorption frequency, we have $Z=1.001-12.961 i$, which is noted to match the value required by equation (5), $Z_{0}\left[1-i \cot \left(k_{0} s\right)\right]=$ $1-16.342 i$, reasonably well. Furthermore, from the impedance for the composite system comprising the DMR and the air layer, one can calculate the reflection as $R=\left(Z_{0}-Z_{c}\right) /$ $\left(Z_{0}+Z_{c}\right)$. The absorption coefficient can then be evaluated as $1-|R|^{2}$, which is plotted as the black curve in Figure $5 \mathrm{c}$. Excellent agreement with the experiment (red circles) is seen.

In Figure $4 \mathrm{~b}$ we have plotted the calculated magnitude of the deformation component $\sqrt{\left\langle(\delta W)^{2}\right\rangle} / W_{s}$ as a function of $\Delta \bar{\omega}$. In agreement with our expectation, the magnitude of the deformation component is seen to diverge at $\tilde{\omega}(\Delta \bar{\omega}=0)$, at which point both the required $s$ and $\beta$ approach zero. To confirm the large deformation component in this hybrid resonance, we have used laser vibrometer to measure the vibrational profile of the DMR at the total absorption frequency. The experimental result, normalized to $1 \mathrm{~Pa}$ (corresponding to $94 \mathrm{~dB}$ ) of incident sound amplitude, is shown in Figure 3c as red circles. This hybrid resonance profile is clearly a linear superposition of the membrane's two eigenmodes (Figure 3b), and resembles the motion profile at DMR's anti-resonance [2]. In the same figure are shown the numerical simulation results (black curve), which are in excellent agreement with the measured profile. From the measured displacement, we can evaluate $\langle W\rangle \simeq 2.8 \mu \mathrm{m}$, which is close to the incident sound amplitude of $W_{s}=2.5 \mu \mathrm{m}$ - a clear evidence of impedance matching. But $\langle W\rangle$ is noted to be one order of magnitude smaller than the maximum displacement of the platelet (over $40 \mu \mathrm{m}$ ). We can also deduce from the measured data that $\sqrt{\left\langle(\delta W)^{2}\right\rangle}=5.2\langle W\rangle \simeq 14.5 \mu \mathrm{m}$, which is consistent with the relation shown in Figure $4 b$. This significantly amplified displacement component near the anti-resonance frequency explains the total absorption functionality for the weakly lossy membrane in subwavelength.

\section{Concluding remarks}

In this work we use a framework that is based on the geometric perspective to derive upper bounds on absorption by thin membrane-type resonators. It is seen that the CPA and hybrid resonance constitute two scenarios in which perfect absorption can be achieved. In particular, the hybrid resonance utilizes multiple scatterings generated by a back reflecting surface to create a new resonance whose frequency is in the vicinity of the anti-resonance. The hybrid resonance's very large deformation component of the membrane, which is nonradiative, is seen to couple with impedance matching to yield perfect absorption. All theoretical predictions are shown to be in excellent agreement with experimental observations.

Acknowledgements. This work is supported by Hong Kong RGC Grants AoE/P-02/12.

\section{References}

1. L. Landau, E. Lifshitz, Theory of elasticity, Pergamon Press, 1970, Chap. 5, p. 153

2. Z. Yang, J. Mei, M. Yang, N. Chan, P. Sheng, Phys. Rev. Lett. 101 (2008) 204301.

3. S.H. Lee, C.M. Park, Y.M. Seo, C.K. Kim, Phys. Rev. B 81 (2010) 241102.

4. C.M. Park, J.J. Park, S.H. Lee, Y.M. Seo, C.K. Kim, S.H. Lee, Phys. Rev. Lett. 107 (2011) 194301.

5. J.J. Park, K. Lee, O.B. Wright, M.K. Jung, S.H. Lee, Phys. Rev. Lett. 110 (2013) 244302.

6. M. Yang, G. Ma, Z. Yang, P. Sheng, Phys. Rev. Lett. 110 (2013) 134301.

7. G. Ma, M. Yang, Z. Yang, P. Sheng, Appl. Phys. Lett. 103 (2013) 011903.

8. J. Mei, G. Ma, M. Yang, Z. Yang, W. Wen, P. Sheng, Nat. Commun. 3 (2012) 756.

9. M. Yang, Y. Li, C. Meng, C. Fu, J. Mei, Z. Yang, P. Sheng, C. R. Mecanique 343 (2015) 635-644.

10. G. Ma, M. Yang, S. Xiao, Z. Yang, P. Sheng, Nat. Mater. 13 (2014) 873. 
11. M. Yang, G. Ma, Y. Wu, Z. Yang, P. Sheng, Phys. Rev. B 89 (2014) 064309.

12. Y. Chong, L. Ge, H. Cao, A.D. Stone, Phys. Rev. Lett. 105 (2010) 053901.

13. W. Wan, Y. Chong, L. Ge, H. Noh, A.D. Stone, H. Cao, Science 331 (2011) 889.

14. S. Li, J. Luo, S. Anwar, S. Li, W. Lu, Z.H. Hang, Y. Lai, B. Hou, M. Shen, C. Wang, Sci. Rep. 4 (2014) 7369.
15. J.R. Piper, S. Fan, ACS Photonics 1 (2014) 347.

16. P. Wei, C. Croënne, S.T. Chu, J. Li, Appl. Phys. Lett. 104 (2014) 121902.

17. X. Cai, Q. Guo, G. Hu, J. Yang, Appl. Phys. Lett. 105 (2014) 121901.

18. M. Yang, C. Meng, C. Fu, Y. Li, Z. Yang, P. Sheng, Appl. Phys. Lett. 107 (2015) 104104.

Cite this article as: Yang M, Ma G, Yang Z \& Sheng P: Subwavelength perfect acoustic absorption in membrane-type metamaterials: a geometric perspective. EPJ Appl. Metamat. 2015, 2, 10. 